\title{
Galaxy rotation curves and preferred reference frame effects
}

\author{
Hossein Ghaffarnejad $^{\mathrm{a}} \mathbb{D}$, Razieh Dehghani ${ }^{\mathrm{b}}$ \\ Faculty of Physics, Semnan University, Semnan 35131-19111, Iran
}

Received: 27 September 2018 / Accepted: 25 May 2019 / Published online: 4 June 2019

(C) The Author(s) 2019

\begin{abstract}
As an alternative to dark matter models we use generalized Jordan-Brans-Dicke scalar-vector-tensor (JBD-SVT) gravity model to study the behavior of the rotational velocities of test particles moving around galaxies. To do so we consider an interaction potential $U\left(\phi, N_{\mu}\right)$ between the Brans-Dicke scalar field $\phi$ and time like dynamical fourvector field $N_{\mu}$ which plays as four velocity of a preferred reference frame. We show that at in weak field limits metric solution of the galaxy under consideration reaches to a modified Schwarzschild-de Sitter space in which mass of the vector field plays as an effective cosmological constant. In fact the present work proposes modification on the formulation of Newton's gravitational acceleration. This is used to explain circular velocity of galaxies without postulating dark matter. We also check our theoretical results with empirical baryonic Tully Fisher relation which states a linear relations between the rotational speed of galaxies and their mass. Mathematical calculation predict a good correspondence between our theoretical results and experimental observations for a set of 12 spiral galaxies.
\end{abstract}

\section{Introduction}

Observations of the dynamics of galaxies as well as the dynamics of the whole Universe indicates that a main part of the Universe's mass must be missing. According to the works done by authors in Ref. [1], this missing mass is possibly made by (the unknown) dark matter. So galactic scale dynamics is one of the important systems which is subjected to the dark matter studies. The observations of galaxies show that there is a discrepancy between the observed dynamics and the mass inferred from luminous matter [2,3]. Since no dark matter has been detected so far, replacing dark matter by a modified gravity theory, is an alternative approach to the

\footnotetext{
a e-mail: hghafarnejad@semnan.ac.ir

b e-mail: Razieh-Dehghani@semnan.ac.ir
}

problem of missing mass [4]. There are different approaches to solve this problem, such as Modified Newtonian Dynamics (MOND) [5] and it's relativistic extension [6], Modified Gravity (MOG) or its generally covariant version that called scalar-vector-tensor gravity theory (STVG) [4], conformal gravity [7], nonsymmetric gravity theory (NGT) [8] and nonlocal gravity $[9,10]$. In STVG theory the dynamics of a test particle is given by a modified equation of motion. Since the metric field is coupled to scalar fields and a massive vector field, the solution of the field equations for a point mass is different from the point mass Schwarzschild solution of general relativity [11]. The predictions of STVG theory for the rotation curves of galaxies have been compared to observational data [12], by using a static spherically symmetric point mass metric solution for galaxies under consideration. The same approach has also been applied to the dynamics of globular clusters and clusters of galaxies [13,14]. According to the works done by authors in Refs. [15-20] a new scalarvector-tensor gravity theory is introduced where a time-like dynamical vector field can change a Lorentzian signature of the background to an Euclidian form. The dynamical vector field is usually considered as four velocity of preferred reference frames. Another approach was used to extend the Jordan-Brans-Dicke scalar-tensor gravity theory [21] and called as Jordan-Brans-Dicke scalar-vector-tensor (JBDSVT) model by one of the authors in Refs. [22,23]. Several applications of this model are studied in Refs. [24-27]. The main motivations to present this JBD-SVT gravity model are as follows: the exact Lorentz invariance is impossible to test uniformly, as the boost parameter of this group is unrestrained. It also leads to the problem of divergences in quantum field theory associated with states of arbitrarily high energy and momentum [22]. This problem can be solved by a short distance high energy cutoff length which violates the Lorentz invariance [19]. Lorentz invariance violation causes to change the metric signature of space-time. For these reasons, we scrutinize the possibility that there is a preferred rest frame at each space-time point. If this frame was to be a fixed 
structure, it would violate general covariance, then the matter energy-momentum tensor is not divergence-less and the Einstein field equations is inconsistent. In order to preserve general covariance, the preferred frame should be considered dynamical. In this case dynamical frame could be defined by a vector field [17,28-30] or by the gradient of a scalar field $[31,32]$. One of the remarkable results of a suitable dynamical preferred frame, is to have two metrics with inequivalent causal structure named as Lorentzian and Euclidean metrics, respectively. The quantum cosmology viewpoint of the very early universe models describe its origin as a quantum tunneling from Euclidian to the Lorentzian space-time [22]. In both of them the space-time coordinates are still real coordinates. Whereas an ordinary way to obtain an Euclidean metric solution of the Einstein field equations, is to introduce a complex time variable $\tau=i t$, so the Lorentzian signature of the metric is changed to its Euclidean version [22].

In accordance with the works has been done previously $[4,7-10]$, in the weak field approximation the potential for a matter distribution of an extended object behaves like the Newtonian potential with an enhanced gravitational constant and an additional Yukawa potential. Applying a general potential $U\left(\phi, N_{\mu}\right)$ and weak field approximation of the background metric obtained from dynamical field equations of the JBD-SVT gravity model [22] we study rotation curves of galaxies without postulating exotic dark matter which usually is described by dynamical scalar fields. This paper is organized as follows.

In Sect. 2 we defined the JBD-SVT gravity [22] and calculate weak field limit of the Lagrangian density of the model for spherically symmetric state metric equation. Then we obtained linear order solutions of the Euler-Lagrange equations of the fields. In Sect. 3 the acceleration equation and rotational velocity of a test particle is calculated in the weak field limit. Section 4 is devoted to the observational tests of the model. It is shown that the model is consistent with observational rotation curves of a sample of 12 spiral galaxies and the empirical Tully-Fisher relation. Last section denotes to concluding remarks and outlook of the work.

\section{The model}

Let us start with the following JBD-SVT gravity model [22]

$I_{T}=I_{B D}+I_{N}$

in which

$I_{B D}=\frac{1}{16 \pi} \int d^{4} x \sqrt{-g}\left\{\phi R-\frac{\omega}{\phi} g^{\mu \nu} \nabla_{\mu} \phi \nabla_{\nu} \phi\right\}$

is JBD scalar-tensor gravity [21]. $g$ is absolute value of determinant of the metric tensor $g_{\mu \nu}$ where we use the metric signature convention as $(-,+,+,+) . \phi$ is the Brans-Dicke scalar field and $\omega$ is the Brans-Dicke adjustable coupling constant. The second term of the action (2.1) is

$$
\begin{aligned}
I_{N}= & \frac{1}{16 \pi} \int d^{4} x \sqrt{-g}\left\{\zeta\left(x^{\nu}\right)\left(g^{\mu \nu} N_{\mu} N_{v}+1\right)+2 \phi F_{\mu \nu} F^{\mu \nu}\right. \\
& +U\left(\phi, N_{\mu}\right)-\phi N_{\mu} N^{v}\left(2 F^{\mu \lambda} \Omega_{\nu \lambda}+F^{\mu \lambda} F_{\nu \lambda}\right. \\
& \left.\left.+\Omega^{\mu \lambda} \Omega_{v \lambda}-2 R_{v}^{\mu}+\frac{2 \omega}{\phi^{2}} \nabla^{\mu} \phi \nabla_{\nu} \phi\right)\right\}
\end{aligned}
$$

for which

$F_{\mu \nu}=2\left(\nabla_{\mu} N_{\nu}-\nabla_{\nu} N_{\mu}\right), \quad \Omega_{\mu \nu}=2\left(\nabla_{\mu} N_{\nu}+\nabla_{\nu} N_{\mu}\right)$

describes action of a unit time-like dynamical four vector field $N_{\mu}$. In other words it is the action of a preferred reference frame with four velocity $N_{\mu}\left(x^{\nu}\right)$. Up to the terms of $\zeta\left(x^{\nu}\right)$ and the scalar potential $U\left(\phi, N_{\mu}\right)$, the action (2.3) is obtained by transforming the JBD action (2.2) under the metric transformation $g_{\mu \nu} \rightarrow g_{\mu \nu}+2 N_{\mu} N_{\nu}$. One can follow Ref. [22] for more details. Action (2.3) shows that the vector field $N_{\mu}$ is coupled non-minimally to the JBD scalar field $\phi$ and the metric field $g_{\mu \nu}$. Action (2.1) is written in units $c=G=\hbar=1$. The undetermined Lagrange multiplier $\zeta\left(x^{v}\right)$ controls $N_{\mu}$ to be an unit time-like vector field. $\phi$ describes inverse of variable Newton's gravitational coupling parameter and its dimension is (length) $)^{-2}$ in units $c=G=\hbar=1$. Present limits of dimensionless BD parameter $\omega$ based on time-delay experiments [33-36] requires $\omega \geq 4 \times 10^{4}$. General relativistic approach of the BD gravity action (2.2) is obtained by setting $\omega \rightarrow \infty$ [37] for which one can infer

$$
\lim _{\omega \rightarrow \infty} \phi \approx \frac{1}{G}
$$

where $G$ is the Newtonian coupling constant.

Let us consider a general spherically symmetric static metric equation which can be expressed in the following isotropic form [21]

$$
\begin{aligned}
d s^{2}= & -e^{2 \epsilon \alpha(r)} d t^{2} \\
& +e^{2 \epsilon \beta(r)}\left[d r^{2}+r^{2} d \theta^{2}+r^{2} \sin ^{2} \theta d \varphi^{2}\right]
\end{aligned}
$$

where $\epsilon$ is a constant. In order to study the behavior of JBD-SVT in astrophysical scales we should apply weak field approximation for the dynamics of the fields by perturbing the fields around Minkowski spacetime. In this case $\epsilon$ should have small values and will be order parameter of the perturbation. Furthermore, $\epsilon$ should be defined versus the parameter of the model which comes from non-linear counterpart of the action. In the BD action (2.2), $\omega$ determines nonlinear counterpart of the action. Hence it is suitable to choose $\epsilon=\epsilon(\omega)$. One can write perturbation series form of the fields which up to the second order terms are

$e^{2 \epsilon \alpha(r)} \approx 1+2 \epsilon \alpha(r)+O\left(\epsilon^{2}\right)$ 
$e^{2 \epsilon \beta(r)} \approx 1+2 \epsilon \beta(r)+O\left(\epsilon^{2}\right)$,

and

$\phi(r) \approx \frac{1}{G}\left[1+\epsilon \psi(r)+O\left(\epsilon^{2}\right)\right]$.

For static spherically symmetric metric (2.6) the vector field $N_{\mu}$ and the tensor fields $F_{\mu \nu}$ and $\Omega_{\mu \nu}$ should depend on the radial coordinate $r$ for which one can choose [38]

$N_{\mu}(r)=(b(r), q(r), 0,0)$

where the time like condition $g^{\mu \nu} N_{\mu} N_{\nu}=-1$ in weak field limits reads

$-b^{2}(r)+q^{2}(r) \approx-1$.

Substituting (2.10) into (2.4) we obtain

$F_{t r}(r)=-2 b^{\prime}(r), \quad \Omega_{t r}(r)=2 b^{\prime}(r)-4 \epsilon b(r) \alpha^{\prime}(r)$,

and

$$
\Omega_{t t} \approx-4 \epsilon q \alpha^{\prime}, \quad \Omega_{r r} \approx 4 q^{\prime}-4 \epsilon q \beta^{\prime}
$$

where prime denotes to derivative with respect to $r$ coordinate and we used linear order terms of non-vanishing Christoffel symbols $\Gamma_{t t}^{r}(r)=\Gamma_{t r}^{t}(r) \approx \epsilon \alpha^{\prime}(r)$ and $\Gamma_{r r}^{r} \approx \epsilon \beta^{\prime}(r)$ for small $\epsilon$. Substituting (2.7) and (2.8) we obtain $t t$ and $r r$ components of the Ricci tensor for small $\epsilon$ as

$$
\begin{aligned}
& R_{t t} \approx-\frac{\epsilon}{r}\left[r \alpha^{\prime \prime}+2 \alpha^{\prime}\right]+O\left(\epsilon^{2}\right), \\
& R_{r r} \approx \frac{\epsilon}{r}\left[2 \beta^{\prime}+r \alpha^{\prime \prime}+2 r \beta^{\prime \prime}\right]+O\left(\epsilon^{2}\right)
\end{aligned}
$$

where the 4D Ricci scalar $R_{\mu}^{\mu}$ reads

$R \approx \frac{2 \epsilon}{r}\left[2 \alpha^{\prime}+4 \beta^{\prime}+r \alpha^{\prime \prime}+2 r \beta^{\prime \prime}\right]+O\left(\epsilon^{2}\right)$.

Substituting the above perturbative functions into the $\mathrm{BD}$ action (2.2) we obtain $I_{B D}=\int d t d r \mathcal{L}_{B D}$ in which $\mathcal{L}_{B D}$ is the Brans Dicke Lagrangian density such that

$$
\begin{aligned}
\mathcal{L}_{B D}= & \frac{\epsilon}{G}\left[r \alpha^{\prime}+2 r \beta^{\prime}+\frac{r^{2} \alpha^{\prime \prime}}{2}+r^{2} \beta^{\prime \prime}\right] \\
& +\frac{\epsilon^{2}}{G}\left[(\alpha+3 \beta+\psi)\left[r \alpha^{\prime}+2 r \beta^{\prime}+\frac{r^{2} \alpha^{\prime \prime}}{2}+r^{2} \beta^{\prime \prime}\right]\right. \\
& \left.-\frac{\omega}{4} r^{2} \psi^{\prime 2}\right]
\end{aligned}
$$

Integrating by part, we remove $\alpha^{\prime \prime}$ and $\beta^{\prime \prime}$ terms of the Lagrangian density (2.16) to obtain its effective counterpart which up to third order term $O\left(\epsilon^{3}\right)$ is as follows

$$
\begin{aligned}
\mathcal{L}_{B D}^{e f f}= & -\frac{\epsilon^{2} r^{2}}{G}\left[\frac{\omega \psi^{\prime 2}}{4}+\frac{\alpha^{\prime 2}}{2}+3 \beta^{\prime 2}\right. \\
& \left.+\frac{5}{2} \alpha^{\prime} \beta^{\prime}+\frac{\alpha^{\prime} \psi^{\prime}}{2}+\beta^{\prime} \psi^{\prime}\right] .
\end{aligned}
$$

If we use similar calculations for the action functional (2.3) then we will have $I_{N}=\int d t d r \mathcal{L}_{N}$ where $\mathcal{L}_{N}$ is the Lagrangian density of the non-minimal interacting vector field which is defined by

$\mathcal{L}_{N}=\mathcal{L}_{N}^{(0)}+\epsilon \mathcal{L}_{N}^{(1)}+\epsilon^{2} \mathcal{L}_{N}^{(2)}+\cdots$

where

$$
\begin{aligned}
\mathcal{L}_{N}^{(0)}= & \frac{r^{2}}{G}\left(-10 b^{\prime 2}+\frac{V_{0}(b)}{4}\right) \\
\mathcal{L}_{N}^{(1)}= & \frac{r^{2}}{G}\left\{\frac{V_{1}}{4}+\frac{(\alpha+3 \beta) V_{0}}{4}+[14 \beta+2 \alpha-10 \psi\right. \\
& \left.+12 b^{2}(\alpha-\beta)\right] b^{\prime 2}-\frac{\alpha^{\prime \prime}}{2}+\left(b^{2}-1\right) \beta^{\prime \prime} \\
& +\frac{\left(b^{2}-1\right) \beta^{\prime}}{r}-\frac{b^{2} \alpha^{\prime}}{r}+8 b\left(1-b^{2}\right) b^{\prime} \alpha^{\prime} \\
& \left.+4 b\left(3-2 b^{2}\right) b^{\prime} \beta^{\prime}\right\} \\
\mathcal{L}_{N}^{(2)}= & \frac{r^{2}}{G}\left\{\frac{V_{2}}{4}+\frac{(\alpha+3 \beta) V_{1}}{4}+16 b^{2}\left(b^{2}-1\right) \alpha^{\prime} \beta^{\prime}\right. \\
& -8\left(b^{2}-1\right)^{2} \beta^{\prime 2}-8 b^{4} \alpha^{\prime 2}+\frac{\left(1-b^{2}\right) \omega \psi^{\prime 2}}{2} \\
& +\left(b^{2}-1\right)(\alpha-\beta+\psi) \beta^{\prime \prime} \\
& +\left[\frac{(\beta-\alpha-\psi)}{2}+2 b^{2}(\alpha-\beta)\right] \alpha^{\prime \prime} \\
& +\frac{(\alpha-\beta)\left(b^{2}-1\right) \beta^{\prime}}{r}+\frac{\left[b^{2}(\alpha-3 \beta)-1\right] \alpha^{\prime}}{r} \\
& +[4(\alpha+3 \beta)(3 \alpha+4 \beta)+2 \psi(5 \alpha+\beta) \\
& \left.+12 b^{2}(\alpha-\beta)(\alpha+3 \beta-\psi)\right] b^{\prime 2}+4[8 \beta+3 \psi \\
& \left.+2 b^{2}(5 \beta-3 \alpha-\psi)\right] b b^{\prime} \alpha^{\prime}+4[\alpha+29 \beta-2 \psi \\
& \left.\left.+b^{2}(2 \psi-22 \beta+2 \alpha)\right] b b^{\prime} \beta^{\prime}\right\} \\
& +2.21)
\end{aligned}
$$

Integrating by part and removing $\alpha^{\prime \prime}$ and $\beta^{\prime \prime}$, the effective counterpart of equations (2.20) and (2.21) will have the form

$$
\begin{aligned}
\mathcal{L}_{N}^{(1) e f f}= & \frac{r^{2}}{G}\left\{\frac{V_{1}}{4}+\frac{(\alpha+3 \beta) V_{0}}{4}+[14 \beta+2 \alpha-10 \psi\right. \\
& \left.+12 b^{2}(\alpha-\beta)\right] b^{\prime 2}-\frac{\left(b^{2}-1\right) \beta^{\prime}}{r}+\frac{\left(1-b^{2}\right) \alpha^{\prime}}{r} \\
& \left.+8 b\left(1-b^{2}\right) b^{\prime} \alpha^{\prime}+2 b\left(5-4 b^{2}\right) b^{\prime} \beta^{\prime}\right\}
\end{aligned}
$$

and

$$
\begin{aligned}
\mathcal{L}_{N}^{(2) e f f}= & \frac{r^{2}}{G}\left\{\frac{V_{2}}{4}+\frac{(\alpha+3 \beta) V_{1}}{4}\right. \\
& +\left(16 b^{4}-16 b^{2}+\frac{1}{2}\right) \alpha^{\prime} \beta^{\prime} \\
& +\left(9-8 b^{2}\right)\left(b^{2}-1\right) \beta^{\prime 2} \\
& -\left(b^{2}-1\right) b^{\prime} \psi^{\prime}+\frac{\left(1-b^{2}\right) \omega \psi^{\prime 2}}{2}
\end{aligned}
$$




$$
\begin{aligned}
& +\frac{\left[\psi+\alpha-\beta-1+b^{2}(\beta-3 \alpha)\right] \alpha^{\prime}}{r} \\
& +\frac{\alpha^{\prime} \psi^{\prime}}{2}+\frac{(\beta-\alpha-2 \psi)\left(b^{2}-1\right) \beta^{\prime}}{r} \\
& +[4(\alpha+3 \beta)(3 \alpha+4 \beta)+2 \psi(5 \alpha+\beta) \\
& \left.+12 b^{2}(\alpha-\beta)(\alpha+3 \beta-\psi)\right] b^{\prime 2}+4[9 \beta+3 \psi \\
& \left.-\alpha+2 b^{2}(5 \beta-3 \alpha-\psi)\right] b b^{\prime} \alpha^{\prime} \\
& +\left(\frac{1}{2}-2 b^{2}-8 b^{4}\right) \alpha^{\prime 2}+2[\alpha+59 \beta-5 \psi \\
& \left.\left.+4 b^{2}(\psi-11 \beta+\alpha)\right] b b^{\prime} \beta^{\prime}\right\} .
\end{aligned}
$$

Without reducing the generality of the issue, we assume that the interaction potential has the following form

$$
\begin{aligned}
G U\left(\phi, N^{\mu}\right) \approx & V_{0}(b)+\epsilon V_{1}\left(\phi, N_{\mu}\right) \\
& +\epsilon^{2} V_{2}\left(\phi, N_{\mu}\right)+O\left(\epsilon^{3}\right)
\end{aligned}
$$

Adding (2.17), (2.19), (2.22) and (2.23) we can obtain total effective Lagrangian density of the system as follows

$G \mathcal{L}_{\text {tot }}^{\text {eff }}=L_{\text {tot }}^{(0)}+\epsilon L_{\text {tot }}^{(1)}+\epsilon^{2} L_{\text {tot }}^{(2)}+O\left(\epsilon^{3}\right)$

where

$$
\begin{aligned}
L_{\text {tot }}^{(0)}= & r^{2}\left(\frac{V_{0}}{4}-10 b^{\prime 2}\right) \\
L_{\text {tot }}^{(1)}= & r^{2}\left\{\frac{V_{1}}{4}+\frac{(\alpha+3 \beta) V_{0}}{4}+[14 \beta+2 \alpha-10 \psi\right. \\
& \left.+12 b^{2}(\alpha-\beta)\right] b^{\prime 2}-\frac{\left(b^{2}-1\right) \beta^{\prime}}{r}+\frac{\left(1-b^{2}\right) \alpha^{\prime}}{r} \\
& \left.+8 b\left(1-b^{2}\right) b^{\prime} \alpha^{\prime}+2 b\left(5-4 b^{2}\right) b^{\prime} \beta^{\prime}\right\}
\end{aligned}
$$

and

$$
\begin{aligned}
L_{\text {tot }}^{(2)}= & r^{2}\left\{\frac{V_{2}}{4}+\frac{(\alpha+3 \beta) V_{1}}{4}+\left(16 b^{4}-16 b^{2}-2\right) \alpha^{\prime} \beta^{\prime}\right. \\
& -\left(8 b^{4}-17 b^{2}+12\right) \beta^{\prime 2}-\left(b^{2}-1\right) b^{\prime} \psi^{\prime} \\
& +\frac{\left(1-2 b^{2}\right) \omega \psi^{\prime 2}}{4}+\frac{\left[\psi+\alpha-\beta-1+b^{2}(\beta-3 \alpha)\right] \alpha^{\prime}}{r} \\
& -\beta^{\prime} \psi^{\prime}+\frac{(\beta-\alpha-2 \psi)\left(b^{2}-1\right) \beta^{\prime}}{r} \\
& +[4(\alpha+3 \beta)(3 \alpha+4 \beta)+2 \psi(5 \alpha+\beta) \\
& \left.+12 b^{2}(\alpha-\beta)(\alpha+3 \beta-\psi)\right] b^{\prime 2}+4[9 \beta+3 \psi \\
& \left.-\alpha+2 b^{2}(5 \beta-3 \alpha-\psi)\right] b b^{\prime} \alpha^{\prime}-2 b^{2}\left(1+4 b^{2}\right) \alpha^{\prime 2} \\
& \left.+2\left[\alpha+59 \beta-5 \psi+4 b^{2}(\psi-11 \beta+\alpha)\right] b b^{\prime} \beta^{\prime}\right\}
\end{aligned}
$$

for

$V_{1}=0, \quad V_{2}=0$.

The effective total lagrangian (2.25) shows that in the weak field limit and slowly varying Brans-Dicke scalar field where $\epsilon(\omega) \rightarrow 0$ and $\omega \rightarrow \infty$ the lagrangian density of the vector field is dominated instead of the Brans Dicke scalar field lagrangian density. In fact for $\omega \rightarrow \infty$ the BransDicke action (2.2) reaches to the Einstein-Hilbert counterpart which can not support the galactic rotation curves alone. In the weak field limits $(\epsilon \rightarrow 0)$ one can see that the Brans Dicke Lagrangian counterpart (2.17) vanishes and so the vector field lagrangian density (2.26) is dominated to determine the galactic rotation curves at $\epsilon=0$. Now we obtain Euler lagrange equations of the fields $b(r), \alpha(r), \beta(r)$ and $\psi(r)$ by varying the total Lagrangian density (2.25) and solve them order by order as follows. Setting $\epsilon=0$ the zero order term of the Euler-Lagrange equation reads

$b^{\prime \prime}+\frac{2 b^{\prime}}{r}+\frac{1}{80} \frac{\partial V_{0}(b)}{\partial b}=0$

which is independent of other fields and so can be solved alone. To solve the above equation we should choose some suitable potentials for which solutions of the above equation satisfy the time-like condition $q=\sqrt{b^{2}-1}$ (i.e. $|b|>1$ ) with boundary condition $q(\infty)=0$. Thus we choose the following ansatz for the potential $V_{0}(b)$.

$V_{0}(b)=80 \mu^{2} b-40 \mu^{2} b^{2}$

in which $\mu$ can be described as mass of the vector field $N_{\mu}$. We will see that $\mu$ is related with a suitable effective cosmological constant $\Lambda$ which means the Hubble constant of the expansion of the universe. Also the above potential makes the Eq. (2.30) as linear differential equation. We should point that, nonlinear differential equations in general form, have usually unstable solutions which may be reach to chaos. Substituting the above potential into the Eq. (2.30) one can obtain a particular convergent solution for $b(r)$ as follows.

$b(r)=1+A \frac{e^{-\mu r}}{r}$

where $A$ is integral constant which should be considered as fitting parameter when we study its effect on the galactic circular velocity. The Eq. (2.30) with potential (2.31) has other solution as $\frac{e^{\mu r}}{r}$ for $\mu>0$ which we do not consider here because it diverges to infinity at $r \rightarrow \infty$. It is clear that only the decaying exponentials should be a part of the physical solution where the energy does not diverge to some infinite values and as we should retrieve the standard Newtonian gravitational potential at $r \rightarrow \infty$. Substituting (2.32), the equation $q(r)= \pm \sqrt{b^{2}-1}$ reads

$q(r)= \pm \sqrt{\frac{A^{2} e^{-2 \mu r}}{r^{2}}+\frac{2 A e^{-\mu r}}{r}}$

which satisfies the boundary condition $q(\infty)=0$. At large distances $r \rightarrow \infty$ we see the vector field components approach to $(b, q) \approx(1,0)$ and $\left(b^{\prime}, q^{\prime}\right) \approx(0,0)$. Substituting these asymptotic solutions into the effective Lagrangian 
densities (2.26), (2.27) and (2.28) we obtain asymptotic behavior of the total effective Lagrangian density (2.25) as follows

$$
\begin{aligned}
& \lim _{r \rightarrow \infty} G \mathcal{L}_{\text {tot }}^{e f f} \approx 10 \mu^{2} r^{2}[1+\epsilon(\alpha+\beta)] \\
& +\epsilon^{2} r^{2}\left[3 \beta^{\prime 2}-10 \alpha^{\prime 2}-\frac{\omega \psi^{\prime 2}}{4}+\frac{(\psi-2 \alpha-1) \alpha^{\prime}}{r}\right. \\
& \left.-2 \alpha^{\prime} \beta^{\prime}-\psi^{\prime} \beta^{\prime}\right]
\end{aligned}
$$

Varying the Lagrangian density (2.34) with respect to the fields $\psi(r), \beta(r)$ and $\alpha(r)$ we obtain their Euler-Lagrange equations which up to second order terms $O\left(\epsilon^{2}\right)$ become respectively as

$$
\begin{aligned}
& 2 r \alpha^{\prime}+\omega\left(r^{2} \psi^{\prime}\right)^{\prime} \approx 0 \\
& 10 \mu^{2} r^{2}+\epsilon\left[r^{2}(\psi+2 \alpha-6 \beta)^{\prime}\right]^{\prime} \approx 0
\end{aligned}
$$

and

$$
\begin{gathered}
10 \mu^{2} r^{2}+\epsilon\left\{-2 r \alpha^{\prime}+\left[2 r^{2}(10 \alpha+\beta)^{\prime}\right.\right. \\
\left.+(1+2 \alpha-\psi) r]^{\prime}\right\} \approx 0 .
\end{gathered}
$$

To solve the above equations we first substitute $2 r \alpha^{\prime}$ from (2.35) into the Eq. (2.37) and integrate it to obtain

$$
\begin{aligned}
\frac{10}{3} \mu^{2} r^{2} & +\frac{C_{1}}{r}+\epsilon\left[r(\omega \psi+20 \alpha+2 \beta)^{\prime}\right. \\
& +1-\psi+2 \alpha] \approx 0
\end{aligned}
$$

in which $C_{1}$ is integral constant. The Eq. (2.36) can be integrated alone to obtain

$\psi+2 \alpha-6 \beta=C_{3}+\frac{C_{2}}{r}-\frac{5}{3} \mu^{2} r^{2}$.

Now we substitute $\beta$ from the above equation and $r \alpha^{\prime}$ from the Eq. (2.35) into the Eq. (2.38) to obtain

$$
\begin{aligned}
& \frac{10}{3}\left(1+\frac{\epsilon}{3}\right) \mu^{2} r^{2}+\frac{\left(3 C_{1}+\epsilon C_{2}\right)}{3 r}+\epsilon+\frac{\epsilon(1-59 \omega)}{3} r \psi^{\prime} \\
& -\frac{31 \omega \epsilon}{3} r^{2} \psi^{\prime \prime}-\epsilon \psi+2 \epsilon \alpha \approx 0
\end{aligned}
$$

in which $C_{2,3}$ are integral constants. Substituting $\alpha^{\prime}$ from the Eq. (2.37) into the derivative of the above equation we obtain a linear differential equation for the Brans Dicke scalar field $\psi(r)$ as follows.

$$
\begin{aligned}
\psi^{\prime \prime \prime} & +\left(4-\frac{1}{31 \omega}\right) \frac{\psi^{\prime \prime}}{r}+\left(\frac{65}{31}+\frac{2}{31 \omega}\right) \frac{\psi^{\prime}}{r^{2}} \\
& +\frac{\left(3 C_{1}+\epsilon C_{2}\right)}{31 \omega \epsilon} \frac{1}{r^{4}}-\frac{20(3+\epsilon)}{93 \epsilon \omega} \frac{\mu^{2}}{r} \approx 0 .
\end{aligned}
$$

One can solve explicitly the Eqs. (2.41), (2.40) and (2.39) synchronously to obtain exact solutions for the fields $\psi(r)$, $\alpha(r)$ and $\beta(r)$ respectively as follows.

$$
\epsilon \psi(r)=\frac{\left(3 C_{1}+\epsilon C_{2}\right)}{(4+3 \omega)} \frac{1}{r}+\frac{10(3+\epsilon)}{(1+189 \omega)} \frac{\mu^{2} r^{2}}{3}
$$

$$
+\epsilon C_{4} \frac{r^{f_{-}(\omega)}}{f_{-}(\omega)}+\epsilon C_{5} \frac{r^{f_{+}(\omega)}}{f_{+}(\omega)}+\epsilon C_{6}
$$

where $C_{4,5,6}$ are integral constants and we defined

$$
f_{ \pm}(\omega)=-\frac{1}{2}+\frac{1}{62 \omega} \pm \sqrt{589-\frac{434}{\omega}+\frac{1}{\omega^{2}}}
$$

and

$$
\begin{aligned}
\epsilon \alpha(r)= & -K_{1} \mu^{2} r^{2}+\frac{K_{2}}{r}+\epsilon C_{4} \frac{r^{f_{-}(\omega)}}{g_{-}(\omega)} \\
& +\epsilon C_{5} \frac{r^{f_{+}(\omega)}}{g_{+}(\omega)}+\frac{\epsilon\left(C_{6}-1\right)}{2}
\end{aligned}
$$

where we defined

$$
\begin{aligned}
& K_{1}=\frac{5}{9} \frac{\left(378 \epsilon^{2} \omega+2 \epsilon^{2}+954 \epsilon \omega+5 \epsilon-540 \omega-3\right)}{(1+189 \omega)}, \\
& K_{2}=\frac{(1-2 \epsilon)\left(3 C_{1}+\epsilon C_{2}\right)}{6}
\end{aligned}
$$

with

$$
\begin{aligned}
g_{ \pm}(\omega)= & \frac{f_{ \pm}(\omega)}{31 \omega f_{ \pm}^{2}(\omega)+(28 \omega-1) f_{ \pm}(\omega)+3} \\
\epsilon \beta(r)= & K_{3} \mu^{2} r^{2}+\frac{\epsilon\left(2 C_{6}-C_{3}-1\right)}{6}+\frac{K_{4}}{r}+\epsilon C_{4} \frac{r^{f_{-}(\omega)}}{h_{-}(\omega)} \\
& +\epsilon C_{5} \frac{r^{f_{+}(\omega)}}{h_{+}(\omega)}
\end{aligned}
$$

in which we defined

$$
\begin{aligned}
& K_{3}=\frac{5}{9}\left(\frac{3+\epsilon}{1+189 \omega}\right)-\frac{K_{1}}{3}+\frac{5}{18} \\
& K_{4}=\frac{3 C_{1}[7-8 \epsilon+3 \omega(1-2 \epsilon)]-\epsilon C_{2}[209+8 \epsilon+3 \omega(2 \epsilon+53)]}{18(4+3 \omega)}
\end{aligned}
$$

and

$\frac{6}{h_{ \pm}(\omega)}=\frac{1}{f_{ \pm}(\omega)}+\frac{2}{g_{ \pm}(\omega)}$.

Substituting the above solutions into the relations (2.7), (2.8) and (2.9) we obtain respectively

$$
\begin{aligned}
e^{2 \epsilon \alpha(r)} & \sim 1+\epsilon\left(C_{6}-1\right)-2 K_{1} \mu^{2} r^{2}+\frac{2 K_{2}}{r} \\
+2 \epsilon C_{4} \frac{r^{f_{-}(\omega)}}{g_{-}(\omega)}+2 \epsilon C_{5} \frac{r^{f_{+}(\omega)}}{g_{+}(\omega)} & \left.3 C_{3}-1\right) \\
e^{2 \epsilon \beta(r)} & \sim 1+\frac{\epsilon\left(2 C_{6}-C_{3} \mu^{2} r^{2}\right.}{3} \\
+\frac{2 K_{4}}{r} & +2 \epsilon C_{4} \frac{r^{f_{-}(\omega)}}{h_{-}(\omega)}+2 \epsilon C_{5} \frac{r^{f_{+}(\omega)}}{h_{+}(\omega)}
\end{aligned}
$$


and

$$
\begin{aligned}
& G \phi(r) \sim 1+\epsilon C_{6}+\frac{\left(3 C_{1}+\epsilon C_{2}\right)}{(4+3 \omega)} \frac{1}{r} \\
& +\frac{10(3+\epsilon)}{(1+189 \omega)} \frac{\mu^{2} r^{2}}{3}+\epsilon C_{4} \frac{r^{f_{-}(\omega)}}{f_{-}(\omega)}+\epsilon C_{5} \frac{r^{f_{+}(\omega)}}{f_{+}(\omega)}
\end{aligned}
$$

Now we should fix the integral constants of the above solutions by regarding physical boundary conditions.

Appearance of the square term in the above metric potentials as $\mu^{2} r^{2}$ remember us the de Sitter universe at large scale structure of the metric solution where $\mu^{2}$ behaves as an effective cosmological constant $\Lambda>0$. This means that our solutions treat as de Sitter metric at large distances $\mu r>>1$ while at small distances $\mu r<<1$ the inverse distance factor $\frac{1}{r}$ is dominated instead of the term $\mu^{2} r^{2}$ which can be related to the Schwarzschild counterpart of the metric in absence of the terms $C_{4,5}$. We know that the Schwarzschild de Sitter black hole metric with $\Lambda>0$ is $d s^{2}=-\left(1-2 G M / r-\Lambda r^{2} / 3\right) d t^{2}+d r^{2} /(1-2 G M / r-$ $\left.\Lambda r^{2} / 3\right)+r^{2} d \theta^{2}+r^{2} \sin ^{2} \theta d \varphi^{2}$ which is obtained from $G_{\mu \nu}+\Lambda g_{\mu \nu}=0$ and asymptotically reduces to the vacuum de Sitter space at large distances $r>2 G M$. In weak field limit it reads $d s^{2}=-\left(1-2 G M / r-\Lambda r^{2} / 3\right) d t^{2}+(1+$ $\left.2 G M / r+\Lambda r^{2} / 3\right) d r^{2}+r^{2} d \theta^{2}+r^{2} \sin ^{2} \theta d \varphi^{2}$ which can be compared with solutions (2.51) and (2.52) by setting

$2 K_{1} \mu^{2}=\frac{\Lambda}{3}=2 K_{3} \mu^{2}$,

$2 K_{4}=2 G M=-2 K_{2}, \quad C_{6}=C_{3}=1$

where $M$ is total mass of the central black hole of the Schwarzschild de Sitter space time. In the standard $\Lambda C D M$ cosmological model the unknown exotic dark matter/energy is dominated to support the cosmic inflation and it behaves as an effective cosmological constant. Substituting (2.45), (2.48), (2.49) into the condition (2.54) the equation $K_{3}=K_{1}$ reads

$\epsilon_{ \pm}(\omega)=\frac{-(17+3816 \omega) \pm \sqrt{29340144 \omega^{2}+344016 \omega+1009}}{16(1+189 \omega)}$

and the equation $K_{2}+K_{4}=0$ reduces to the following condition.

$\frac{C_{1}}{C_{2}}=\frac{\epsilon[197+150 \omega+8 \epsilon(4+3 \omega)]}{3[19+12 \omega-8 \epsilon(4+3 \omega)]}=S(\omega)$

in which

$C_{2}=\frac{-6 G M}{(1-2 \epsilon)(3 S(\omega)+\epsilon)}$.

Substituting (2.54) into the solutions (2.51), (2.52) and (2.53) we will have

$e^{2 \epsilon \alpha(r)} \sim 1-\frac{\Lambda}{3} r^{2}-\frac{2 G M}{r}$

$$
\begin{gathered}
+2 \epsilon C_{4} \frac{r^{f_{-}(\omega)}}{g_{-}(\omega)}+2 \epsilon C_{5} \frac{r^{f_{+}(\omega)}}{g_{+}(\omega)} \\
e^{2 \epsilon \beta(r)} \sim 1+\frac{\Lambda}{3} r^{2}+\frac{2 G M}{r} \\
+2 \epsilon C_{4} \frac{r^{f_{-}(\omega)}}{h_{-}(\omega)}+2 \epsilon C_{5} \frac{r^{f_{+}(\omega)}}{h_{+}(\omega)}
\end{gathered}
$$

and

$$
\begin{aligned}
& G \phi(r) \sim 1+\epsilon-\frac{6}{(1-2 \epsilon)(4+3 \omega)} \frac{G M}{r}+J(\omega) \Lambda r^{2} \\
& +\epsilon C_{4} \frac{r^{f_{-}(\omega)}}{f_{-}(\omega)}+\epsilon C_{5} \frac{r^{f_{+}(\omega)}}{f_{+}(\omega)}
\end{aligned}
$$

in which we defined

$$
J(\omega)=\frac{3+\epsilon}{378 \epsilon^{2} \omega+2 \epsilon^{2}+954 \epsilon \omega+5 \epsilon-540 \omega-3} .
$$

Now we should say about physical situations of the integral constants $C_{4,5}$. We can show that they are related to a radial length $r_{q f}$ scale which determines quasi flat regions of the space time where

$e^{2 \epsilon \alpha\left(r_{q f}\right)}=1=e^{2 \epsilon \beta\left(r_{q f}\right)}$

for which the Eqs. (2.58) and (2.59) read

$C_{4}=\frac{\left(\frac{1}{h_{+}(\omega)}+\frac{1}{g_{+}(\omega)}\right)\left(\frac{\Lambda r_{q f}^{2}}{3}+\frac{2 G M}{r_{q f}}\right)}{2 \epsilon r_{q f}^{f_{-}(\omega)}\left(\frac{1}{g_{-}(\omega) h_{+}(\omega)}-\frac{1}{g_{+}(\omega) h_{-}(\omega)}\right)}$

and

$C_{5}=-\frac{\left(\frac{1}{h_{-}(\omega)}+\frac{1}{g_{-}(\omega)}\right)\left(\frac{\Lambda r_{q f}^{2}}{3}+\frac{2 G M}{r_{q f}}\right)}{2 \epsilon r_{q f}^{f_{+}(\omega)}\left(\frac{1}{g_{-}(\omega) h_{+}(\omega)}-\frac{1}{g_{+}(\omega) h_{-}(\omega)}\right)}$.

We know from the Eq. (2.5) which the solution (2.60) should not diverge to infinity at $\omega \rightarrow \infty$. In fact for quasi flat regions the solution (2.60) should reach to the boundary condition (2.5) for which we should set

$$
\begin{gathered}
\lim _{\omega \rightarrow \infty} \epsilon-\frac{6}{(1-2 \epsilon)(4+3 \omega)} \frac{G M}{r_{q f}}+J(\omega) \Lambda r_{q f}^{2} \\
+\epsilon C_{4} \frac{r_{q f}^{f_{-}(\omega)}}{f_{-}(\omega)}+\epsilon C_{5} \frac{r_{q f}^{f_{+}(\omega)}}{f_{+}(\omega)} \rightarrow 0
\end{gathered}
$$

It is easy to check for large $\omega$ we will have asymptotically

$$
\begin{aligned}
f_{-}(\omega) & \approx-24.769+\frac{8.9573}{\omega}, \\
f_{+}(\omega) & \approx 23.769-\frac{8.9251}{\omega} \\
g_{-}(\omega) & \approx-\frac{0.0013517}{\omega}, \quad g_{+}(\omega) \approx \frac{0.0013074}{\omega}
\end{aligned}
$$


and

$$
\begin{aligned}
& h_{-}(\omega) \approx-\frac{0.0040552}{\omega}, \quad h_{+}(\omega) \approx \frac{0.0039223}{\omega} \\
& \epsilon_{-}(\omega) \approx-3.0531+\frac{0.0000314}{\omega}, \\
& \epsilon_{+}(\omega) \approx 0.5293+\frac{0.0020788}{\omega} \\
& \frac{C_{1}(\omega)}{G M} \approx 0.15+\frac{0.005}{\omega}, \quad \frac{C_{2}(\omega)}{G M}-46.15-\frac{1.02}{\omega} \\
& J_{-}(\omega) \approx-\frac{0.00075}{\omega}, \quad J_{+}(\omega) \approx \frac{0.049849}{\omega} \\
& C_{4}^{+}=\frac{6112.788719}{\omega}\left(\frac{\frac{\Lambda}{3} r_{q f}^{2}+\frac{2 G M}{r_{q f}}}{r_{q f}^{-24.769+\frac{8.9573}{\omega}}}\right), \\
& \frac{C_{4}^{-}}{C_{4}^{+}} \approx-0.1734 \\
& C_{5}^{+}=-\frac{5912.452201}{\omega}\left(\frac{\frac{\Lambda}{3} r_{q f}^{2}+\frac{2 G M}{r_{q f}}}{r_{q f}^{23.769-\frac{8.9251}{\omega}}}\right), \\
& \frac{C_{5}^{-}}{C_{5}^{+}} \approx-0.1734
\end{aligned}
$$

and

$\mu_{-}^{2} \approx \frac{\Lambda}{3}\left[2.4012+\frac{0.001356}{\omega}\right]$,

$\mu_{+}^{2} \approx \frac{\Lambda}{3}\left[2.4012-\frac{0.08973}{\omega}\right]$.

The above calculations show that for $\omega \rightarrow \infty$ we will have $J(\infty)=0=C_{4,5}(\infty)$ and so the Eq. (2.65) leads to small $\epsilon_{+}(\infty) \approx+0.5$ instead a zero value. This means the choice $\epsilon_{+}$is physical but not $\epsilon_{-}$. In fact, if we substitute $\epsilon_{-}(\infty) \approx-3$ into the Eq. (2.65) then we can result as $\lim _{\omega \rightarrow \infty} G \phi \rightarrow-2$ which is not a physical boundary condition ( $\phi$ is a positive valued field). However for a large $\omega$ we substitute the above approximated equations together with $\epsilon_{+} \approx 0.5293+\frac{0.0020788}{\omega}$ into the Eq. (2.65) to obtain an equation for $r_{q f}$ as follows.

$0.5293 \omega-\frac{490.4471 G M}{r_{q f}}-29.1413 \frac{\Lambda r_{q f}^{2}}{3} \approx 0$

Regarding $\vartheta$ as a free parameter one can obtain the following identities from (2.75).

$\frac{2 G M}{r_{q f}}=0.0022 \omega \cos ^{2} \vartheta, \quad \frac{\Lambda r_{q f}^{2}}{3}=0.0020 \omega \sin ^{2} \vartheta$

It is remarkable that the left side of the above equation shows that for large $\omega$ the quasi flat region $r_{q f}$ should reach to the Schwarzschild radius $2 G M$ of the central black hole of the galaxy under consideration while the right side of them shows that by raising $\omega$ the quasi flat region $r_{q f}$ reach to the cosmological event horizon of the de Sitter vacuum space $\sqrt{\frac{3}{\Lambda}} \approx 1.833 \times 10^{9}($ light year $)=0.5623 \times 10^{9}($ Parsec $)$. In other words for a fixed $\omega$ the left side equation shows that by rasing $r_{q f}$ then $\vartheta \rightarrow \frac{\pi}{2}$. These helps us to obtain an approximated solution for $r_{q f}$ by setting $\vartheta=\frac{\pi}{2}$ in which we have

$r_{q f} \approx 0.23343 \sqrt{\frac{\omega}{\Lambda}}=7.5785 \times 10^{4} \sqrt{\omega}(K p c)$.

At last we are in a position to write metric solutions for large $\omega$ as follows.

$$
\begin{gathered}
e^{2 \epsilon \alpha_{+}(r)} \sim 1-\frac{\Lambda r^{2}}{3}-\frac{2 G M}{r}-1.6 \\
\times 10^{6} \Lambda r_{q f}^{2}\left[\left(\frac{r_{q f}}{r}\right)^{25}+\left(\frac{r}{r_{q f}}\right)^{24}\right]
\end{gathered}
$$

and

$$
\begin{gathered}
e^{2 \epsilon \beta_{+}(r)} \sim 1+\frac{\Lambda r^{2}}{3}+\frac{2 G M}{r}-0.534 \\
\times 10^{6} \Lambda r_{q f}^{2}\left[\left(\frac{r_{q f}}{r}\right)^{25}+\left(\frac{r}{r_{q f}}\right)^{24}\right]
\end{gathered}
$$

In the following section we obtain equation of motion of a test particle orbiting around galaxy in weak limit of the gravitational field.

\section{Modified equations of motion}

For galactic scales studies one can consider a test particle (a star in our consideration) as a tracer of gravitational field. At first approximation we often assume the galaxies are spherically symmetric objects. When we investigate rotation curves at significantly larger radii than the central region of the galaxy, it is justifiable to use a point-like source approximation for any physical model that does not involve an extended dark matter halo. These approximations are surprisingly useful because of the often very large uncertainty in the baryonic mass-to-light ratio (M/L) of many galaxies. We assume a test star with mass parameter $m$ orbiting around a central body and interact with the 4-vector field $N^{\mu}$ for which one can write its action functional as follows

$I_{T P}=-m \int d \tau \sqrt{g_{\mu \nu} V^{\mu} V^{\nu}}+\lambda \int d \tau N_{\mu} V^{\mu}$

where $N_{\mu}=(b(r), q(r), 0,0)$ given by (2.10) is 4-velocity of the preferred reference frame, $\tau$ is the proper time along the world line of the test particle with the dimension of length. $\lambda$ denotes to the interaction coupling constant between the vector field $N^{\mu}$ and the test particle with four velocity $V^{\mu}=$ 
$\frac{d x^{\mu}}{d \tau}$. Varying (3.1) with respect to the coordinates $x^{\mu}$ one can obtain Euler Lagrange equations of the test particle such that

$\frac{d^{2} x^{\mu}}{d \tau^{2}}+\Gamma_{\alpha \beta}^{\mu} \frac{d x^{\alpha}}{d \tau} \frac{d x^{\beta}}{d \tau}=\frac{\lambda}{m} F^{\mu}{ }_{\nu} \frac{d x^{\nu}}{d \tau}$.

For the line element (2.6), the equation of motion (3.2) in weak field approximation for small $\epsilon$ read

$$
\begin{aligned}
& \frac{d^{2} t}{d \tau^{2}}+\frac{2 \epsilon \alpha^{\prime}}{1+2 \epsilon \alpha}\left(\frac{d t}{d \tau}\right)\left(\frac{d r}{d \tau}\right) \\
& \cong \frac{2 \lambda(1-2 \epsilon \alpha(r))}{m} b^{\prime}(r)\left(\frac{d r}{d \tau}\right), \\
& \frac{d^{2} r}{d \tau^{2}}+\frac{\epsilon \beta^{\prime}}{1+2 \epsilon \beta}\left(\frac{d r}{d \tau}\right)^{2}+\frac{\epsilon \alpha^{\prime}}{1+2 \epsilon \beta}\left(\frac{d t}{d \tau}\right)^{2} \\
& -\frac{\left(r+\epsilon r^{2} \beta^{\prime}+2 \epsilon r \beta\right)}{r+2 \epsilon \beta}\left(\frac{d \theta}{d \tau}\right)^{2} \\
& -r \sin { }^{2} \theta \frac{\left(1+\epsilon \beta^{\prime} r+2 \epsilon \beta\right)}{1+2 \epsilon \beta}\left(\frac{d \varphi}{d \tau}\right)^{2} \\
& =\frac{2 \lambda(1-2 \epsilon \beta(r))}{m} b^{\prime}\left(\frac{d t}{d \tau}\right) \\
& \frac{d^{2} \theta}{d \tau^{2}}+\frac{2\left(1+\epsilon r \beta^{\prime}+2 \epsilon \beta\right)}{r+2 \epsilon \beta r}\left(\frac{d \theta}{d \tau}\right)\left(\frac{d r}{d \tau}\right) \\
& -\sin \theta \cos \theta\left(\frac{d \varphi}{d \tau}\right)^{2}=0
\end{aligned}
$$

and

$$
\begin{aligned}
& \frac{d^{2} \varphi}{d \tau^{2}}+\frac{2\left(1+\epsilon r \beta^{\prime}+2 \epsilon \beta\right)}{r+2 \epsilon \beta r}\left(\frac{d \varphi}{d \tau}\right)\left(\frac{d r}{d \tau}\right) \\
& +\cot \theta\left(\frac{d \varphi}{d \tau}\right)\left(\frac{d \theta}{d \tau}\right)=0 .
\end{aligned}
$$

Because of spherically symmetric property of the metric equation, we choose $\theta=\frac{\pi}{2}$ for planar orbit of the test particle which satisfies trivially equation (3.5). Therefore Eq. (3.6) leads to a conserved angular momentum of the test particle as follows

$\frac{d \varphi}{d \tau}=\frac{L}{m(1+2 \epsilon \beta)} \frac{1}{r^{2}}$

in which $L$ is constant angular momentum of the test particle. In the weak field limit, slow motions of the test particle and for circular orbits we can substitute the approximations $\frac{d t}{d \tau} \approx 1$ and $\frac{d r}{d \tau} \approx 0$ into the geodesic Eqs. (3.3) and (3.4). Equation (3.3) vanishes trivially while Eq. (3.4) leads to the acceleration equation of the test particle, which up to second order term $O\left(\epsilon^{2}\right)$ is

$\frac{d^{2} r}{d t^{2}} \approx-\epsilon \alpha^{\prime}+\frac{L^{2}\left[1-4 \epsilon \beta+\epsilon r \beta^{\prime}\right]}{m^{2} r^{3}}+\frac{2 \lambda b^{\prime}[1-2 \epsilon \beta]}{m}$.

To obtain circular stable orbits of test particle (the test star) we should set $\frac{d r^{2}}{d t^{2}}=0$ and $L=m r v(r)$ in the above equation where $v(r)$ is circular velocity of the test particle moving on a circular orbit with constant radius $r$. Regarding the latter conditions the Eq. (3.8) reads

$v^{2}(r)=\frac{-\frac{2 \lambda r b^{\prime}}{m}+\epsilon\left(r \alpha^{\prime}+\frac{4 \lambda r \beta b^{\prime}}{m}\right)}{1+\epsilon\left(r \beta^{\prime}-4 \beta\right)}$.

Substituting (2.32), (2.78) and (2.79) into the above formula we obtain galactic circular velocity as follows.

$v(r) \approx \sqrt{\frac{G M}{r}} \sqrt{\frac{P(r)}{Q(r)}}$

where we defined

$$
\begin{aligned}
P(r)= & +2 \tan ^{2} \vartheta\left[60\left(\frac{r_{q f}}{r}\right)^{24}-57\left(\frac{r}{r_{q f}}\right)^{25}-\left(\frac{r}{r_{q f}}\right)^{3}\right] \\
& +\sigma\left(1+\frac{r}{r_{q f}} \sqrt{0.002 \omega} \sin \vartheta\right) e^{-\frac{r}{r_{q f}} \sqrt{0.002 \omega} \sin \vartheta} \\
& \times\left\{1-0.002 \omega \cos ^{2} \vartheta\left(\frac{r_{q f}}{r}\right)\right. \\
& +0.002 \omega \sin ^{2} \vartheta\left[\frac{3}{2}\left(\frac{r_{q f}}{r}\right)^{25}+\frac{3}{2}\left(\frac{r}{r_{q f}}\right)^{24}\right. \\
& \left.\left.-\left(\frac{r}{r_{q f}}\right)^{2}\right]\right\}
\end{aligned}
$$

and

$$
\begin{aligned}
Q(r)= & 1-0.005 \omega \cos ^{2} \vartheta\left(\frac{r_{q f}}{r}\right) \\
& +0.002 \omega \sin ^{2} \vartheta\left[24\left(\frac{r_{q f}}{r}\right)^{25}\right. \\
& \left.-18\left(\frac{r}{r_{q f}}\right)^{24}-\left(\frac{r}{r_{q f}}\right)^{2}\right]
\end{aligned}
$$

where we used (2.76) and

$\sigma=\frac{2 A \lambda}{G m M}$

It is easy to check that the above formula for the circular velocity reaches to the Newtonian regime $v_{N}=\sqrt{\frac{G M}{r}}$ at the quasi flat regions $r \approx r_{q f}$, if we set

$P\left(r_{q f}\right)=Q\left(r_{q f}\right)$

which reads

$3 \sin ^{4} \vartheta+4\left(\frac{200}{\omega}-1\right) \sin ^{2} \vartheta+1 \approx 0$

for large $\omega$. The Eq. (3.15) has two different solution as

$\sin ^{2} \vartheta_{1} \approx 1-\frac{200}{\omega}+\cdots$ 
and

$\sin ^{2} \vartheta_{2} \approx \frac{1}{3}+\frac{200}{3 \omega}+\cdots$.

$\vartheta_{1}$ takes $\frac{\pi}{2}$ for $\omega \rightarrow \infty$ and so it is not a suitable physical case because $\tan \vartheta$ given in the Eq. (3.11) diverges to a infinite value. Thus we will continue the work by using (3.17) to calculate the galactic circular velocity (3.10) which for large $\omega$ we choose $\sin ^{2} \vartheta_{2} \sim \frac{1}{3}$ where (3.11) and (3.12) read respectively

$$
\begin{aligned}
P(r)= & +60\left(\frac{r_{q f}}{r}\right)^{24}-57\left(\frac{r}{r_{q f}}\right)^{25}-\left(\frac{r}{r_{q f}}\right)^{3} \\
& +\sigma\left(1+\frac{r}{r_{q f}} \sqrt{\frac{\omega}{1500}}\right) e^{-\frac{r}{r_{q f}} \sqrt{\frac{\omega}{1500}}} \\
& \times\left\{1+\frac{\omega}{1500}\left[\frac{3}{2}\left(\frac{r_{q f}}{r}\right)^{25}+\frac{3}{2}\left(\frac{r}{r_{q f}}\right)^{24}\right.\right. \\
& \left.\left.-\left(\frac{r}{r_{q f}}\right)^{2}-2\left(\frac{r_{q f}}{r}\right)\right]\right\}
\end{aligned}
$$

and

$$
\begin{aligned}
Q(r)= & +\frac{\omega}{1500}\left[24\left(\frac{r_{q f}}{r}\right)^{25}-18\left(\frac{r}{r_{q f}}\right)^{24}-\left(\frac{r}{r_{q f}}\right)^{2}\right. \\
& \left.-5\left(\frac{r_{q f}}{r}\right)\right] .
\end{aligned}
$$

It is easy to check

$$
\lim _{r>>r_{q f}} \frac{P(r)}{Q(r)} \simeq\left(\frac{4750}{\omega}\right)\left(\frac{r}{r_{q f}}\right)
$$

and

$$
\lim _{r<<r_{q f}} \frac{P(r)}{Q(r)} \simeq \frac{\sigma}{16} .
$$

The above galactic circular velocity is for a point like source with total mass $M$. Let us now extend the Eq. (3.10) for a mass distribution of spherically symmetric galaxies where the mass function

$M(r)=4 \pi \int_{0}^{r} \rho(\eta) \eta^{2} d \eta$

is the total amount of ordinary visible matter within a sphere of radius $r$ and $\rho(\eta)$ is the density of visible matter of the spherical galaxy contains an inner core at radius $r=r_{c}$. There are different models which are proposed for mass distribution of visible galaxies. According to the model presented in Ref. [12] we consider here a simple power-law mass distribution function as

$M(r)=M\left(\frac{r}{r_{c}+r}\right)^{3 \varsigma}$

where $\varsigma$ is a constant parameter. The values for $\varsigma$ are 1 and 2 for high surface brightness (HSB) and low surface brightness (LSB) \& Dwarf galaxies respectively. This difference is due to the fact that rotation curves of LSB and dwarf galaxies rise more slowly than those of HSB galaxies [39]. $r_{c}$ is radius of the galaxy core. Inside the core $r<r_{c}$ there is a constant mass density for HSB galaxies. For LSB galaxies it is a raising function as $\rho(r) \approx\left(r / r_{c}\right)^{3}$ inside the core $r<r_{c}$ [12]. Well outside the core radius, where $r>>r_{c}$, Eq. (3.23) implies that

$\lim _{r>>r_{c}} M(r)=M$

in which $M$ is total mass of the galaxy under consideration. Numerical values for $r_{c}$ are given in the Table 1 for a set of 12 observed galaxies. They are obtained by fitting the observational data and our solutions for circular velocity of the galaxies called in the Table 1. At last we substitute (3.23) into the circular velocity given by (3.10) to obtain circular velocity for a mass distributed galaxy as follows.

$v(r)=\sqrt{\frac{G M}{r}}\left(\frac{r}{r_{c}+r}\right)^{\frac{3 \zeta}{2}} \sqrt{\frac{P(r)}{Q(r)}}$

where $P(r)$ and $Q(r)$ should be inserted from (3.18) and (3.19). One can show that the Newton's gravity coupling constant $G \simeq 6.67 \times 10^{-11} \mathrm{~m}^{3} / \mathrm{kg} \mathrm{s}^{2}$ can be rewritten as $G=4.3 \times 10^{-6}\left(\frac{k p c}{M_{\text {sun }}}\right)\left(\frac{\mathrm{km}}{\mathrm{s}}\right)^{2}$. In the latter case the galactic circular velocity can be described in units $\mathrm{km} / \mathrm{s}$ if we choose radial distance $r$ in units $k p c$ instead of the meter and the galactic mass $M$ is given as relative mass with respect to the mass of sun as $\left(M / M_{\text {sun }}\right) \times 10^{10}$. Because $v=\sqrt{\frac{G M}{r}} \rightarrow$ $\left(\frac{\mathrm{km}}{\mathrm{s}}\right) \sqrt{4.3 \times 10^{-6}\left(\frac{k p c}{r}\right)\left(\frac{M}{M_{\text {sun }}}\right)}$. To plot numerical diagram of the circular velocity (3.25) we substitute radial distances in units $k p c$, and $G=4.3 \times 10^{-6}$ and $M$ as $\left(M / M_{\text {sun }} \times 10^{10}\right)$ (see Table 1). In the latter case the velocity is described in $(\mathrm{km} / \mathrm{s})$ units and $r$ in units $k p c$. We plot numerical diagrams for the Brans Dicke parameter as $\omega=40,000$ (see Refs. [33-36]). Other parameters given in the above formula called as $r_{q f}, \sigma, r_{c}$ should be considered as fitting parameters when we set result of our theoretical model with observational data given in the Table 1 . We obtained numerical values for the fitting parameters $\left(r_{q f}, \sigma, r_{c}\right)$ and collect them into the Table 1 for different galaxies which we used to study. circular velocity diagrams for 12 observed galaxies are given in the Fig. 1. There are three charts for each galaxy. That is, the experimental data (black dots) and the theoretical results (red dots) of our model and the Newtonian limit (blue dots) of velocity are plotted in terms of distance from the center of the galaxy. The galaxy mass is given from $[5,40]$. There is two components for each galaxy mass which one of them is related to the neutral Hydrogen gases $\mu_{H I}$ surrounds the galaxy and the other is the central core star-like mass $\mu_{\text {star }}$ and so we should use the galactic relative total mass as $M=\mu_{H I}+\mu_{\text {star }}$ (see the Table 1) for each galaxy rotation curve in the Eq. (3.25). 


\section{Spiral galaxy rotation curves and observational data}

Sample selection

We employ new database of SPARC (Spitzer Photometry and Accurate Rotation Curves) [41] to select observed data of circular velocity of a sample of 12 observed galaxies. They can be seen with black dotes in Fig. 1. In fact SPARC is sample of 175 nearby galaxies with new surface photometry at 3.6 $\mu \mathrm{m}$ and high-quality rotation curves from previous studies about the atomic hydrogen $\mathrm{HI}$ which is one of the best kinematical tracer of the gravitational potential in nearby galaxies [42]. In fact it is representing all rotationally supported morphological types of galaxies. To minimize the star-halo degeneracy, the best approach is to use near-infrared surface photometry (K-band or $3.6 \mu \mathrm{m}$ ), which provides the closest proxy to the stellar mass (see [42] and references therein). In short the SPARC is the largest galaxy sample to date with spatially resolved data on the distribution of both stars and gas. In order to test our model, we have therefore considered a sample of 12 galaxies with well-measured rotation curves extracted from Ref. [5] and presented in Table 1.

Model fit to rotation curves of galaxies

To investigate the rotation curves of galaxies within the framework of JBD-SVT gravity, we suppose that there is no actual dark matter, therefore, such a galaxy essentially consists of baryonic matter containing stars and interstellar gas. Hence the observed circular speeds and the Newtonian ones are derived only from the observed mass of the stellar objects and HI (Neutral Hydrogen) components of galaxies. Moreover we ignore dust in our analysis, since the mass of the dust is at most a few percent of the mass of the interstellar matter.

Armed with Eq. (3.25) we are in a position to plots rotation curves for a sample of 12 spiral galaxies after determining the fitting parameters $\sigma, r_{c}, r_{q f}$ (see Table 1). To do so we use the 'Nonlinear Model Fit' (NMF) function in Wolfram Mathematica software. We observe very good agreement between the observed data points and the fitted curve which are shown in Fig. 1. However, we must use a more sophisticated analysis for the general case where we do not have spherical symmetry, which is left for our next work. In fact the latter considerations bring some higher precision on the results. This is done for some spiral galaxies in Ref. [43] but for MOG gravity model. It is worth to mention that for a broad range of even well researched galaxies there is no consensus on galaxy mass among various sources. Since the galaxy mass is not directly measured hence their estimations are based on various galactic models. So it is not surprising such a broad range of estimations on the mass and core radius of galaxies. However references of mass estimations which we have used in this work, are mentioned in Table 1 and extracted from Refs. [5,40].

\section{Baryonic Tully-Fisher relation}

The baryonic Tully-Fisher relation (BTF) is an empirical relation between baryonic mass $M_{B}$ and maximum rotation velocity $v_{\max }$ which may be roughly expressed as

$M \propto v_{\max }^{a}$

where $3.5 \leq a \leq 4$ is obtained from data analysis by applying the different gravity models such as MOG, MOND and etc. [44]. Since we are interested in fitting rotation curves without any dark matter halo, the baryonic mass of

Table 1 Fitting parameters $\left(\sigma, r_{c}, r_{q f}\right)$ for 12 spiral galaxies. Their observed data which are used from [5,40] are the relative stellar baryonic mass $\mu_{\text {stellar }}=\frac{M_{\text {stellar }}}{M_{\text {sun }}} \times 10^{10}$, the relative mass of neutral Hydrogen $\mu_{H I}=\frac{M_{H I}}{M_{\text {sun }}} \times 10^{10}$ in which galactic total mass is $M_{H I}+M_{\text {stellar }}$

\begin{tabular}{lllllll}
\hline Galaxy & Type & $\mu_{\text {star }}$ & $\mu_{H I}$ & $\sigma$ & $r_{c}$ & $r_{q f}$ \\
\hline NGC 247 & LSB & 0.4 & 0.13 & $-1.71138 \times 10^{7}$ & 0.63315 & 0.193396 \\
NGC 1003 & $L S B$ & 0.30 & 0.82 & 48381.3 & 0.520752 & 0.431666 \\
NGC 2403 & LSB & 1.1 & 0.47 & 1.70255 & 0.470889 & 0.416284 \\
UGC 6930 & $L S B$ & 0.42 & 0.31 & -4652.3 & 0.393393 & 0.269871 \\
UGC 6983 & LSB & 0.57 & 0.29 & $7.75637 \times 10^{10}$ & 0.279081 & 0.321622 \\
NGC 300 & $H S B$ & 0.22 & 0.13 & 15468. & 0.979994 & 0.155275 \\
NGC 3992 & HSB & 15.3 & 0.92 & 9790.58 & -0.299233 & 1.33564 \\
NGC 4157 & $H S B$ & 4.83 & 0.79 & $1.35773 \times 10^{7}$ & 0.149683 & 0.793509 \\
NGC 5907 & $H S B$ & 9.7 & 1.1 & $3.89838 \times 10^{7}$ & -0.873617 & 1 \\
NGC 6503 & $H S B$ & 0.83 & 0.24 & 20052.9 & 0.0169275 & 1 \\
NGC 6946 & $H S B$ & 2.7 & 2.7 & 3.2588 & 0.196318 & 0.404241 \\
NGC 2903 & $H S B$ & 5.5 & 0.31 & -11.4416 & 0.198349 & 0.931982 \\
\hline
\end{tabular}


a galaxy contains stellar and gaseous components only such as $M_{B}=M_{\text {star }}+M_{\text {gas }}$. For galaxies whose rotation curves can be resolved, $v_{\max }$ can be chosen as different forms for instance: the maximum observed velocity, the average velocity, or the velocity at a fixed radius where the rotation curves seams flat. In Ref. [45] one can see different choices for $v_{\max }$ which are applicable to study the empirical TullyFisher equation. Since the rotation curves are close to the flat region so all of these choices are effectively equivalent. In order to construct a baryonic Tully-Fisher relation, one needs to estimate the maximum rotation velocity of a galaxy. There are several ways in which this can be done. We choose $v_{\max }$ to be asymptotically value of the Eq. (3.25) at infinity $v\left(r>>r_{q f}\right)$. To do so we substitute (3.20) into the relation (3.25) for which we obtain
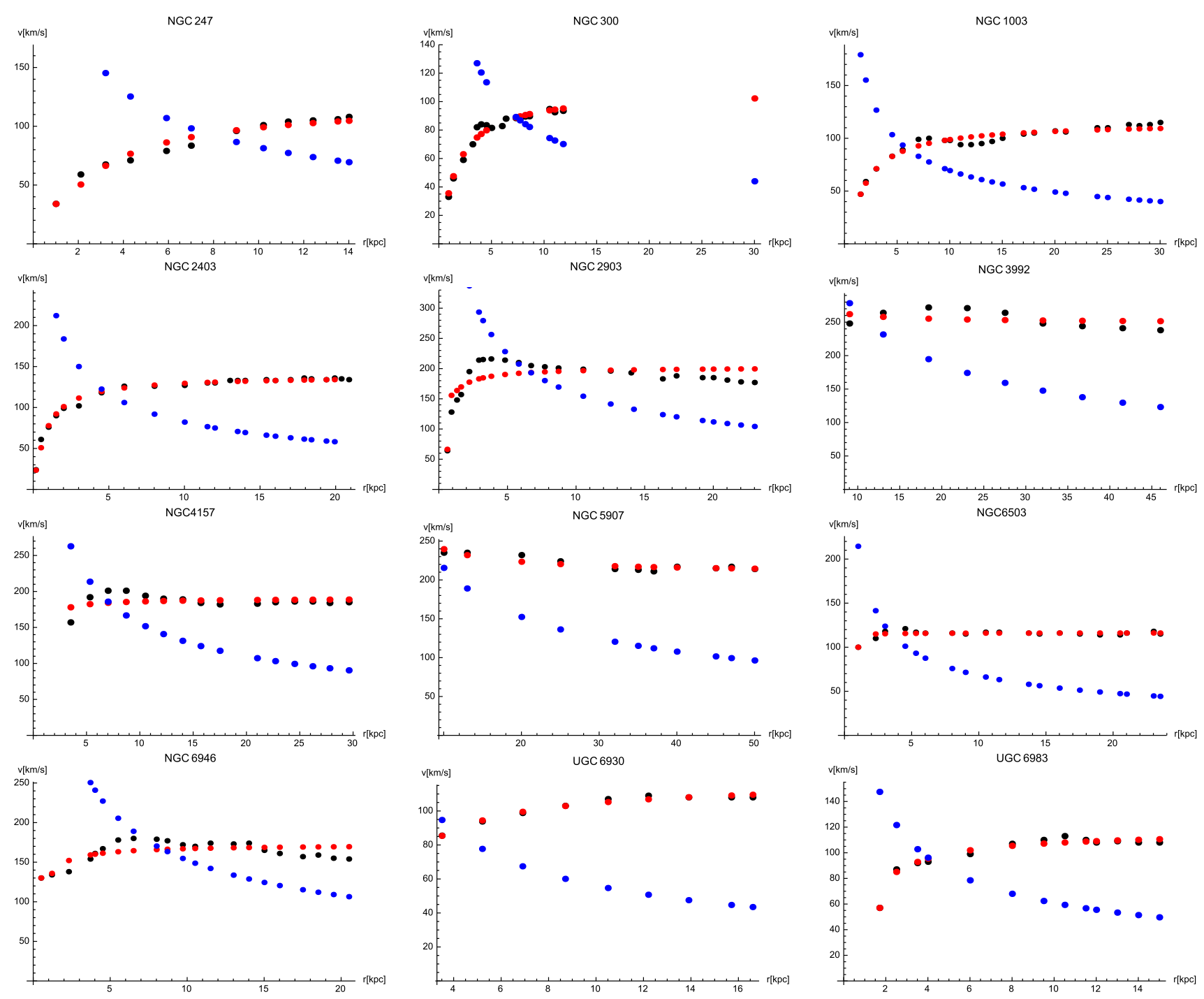

Fig. 1 Galaxy rotation curves for a set of 12 galaxies with different size. Black-dots denote to observational data, red-dots denote to theoretical predictions of our model and blue-dots denote to the Newtonian counterpart of the galactic rotational curves 

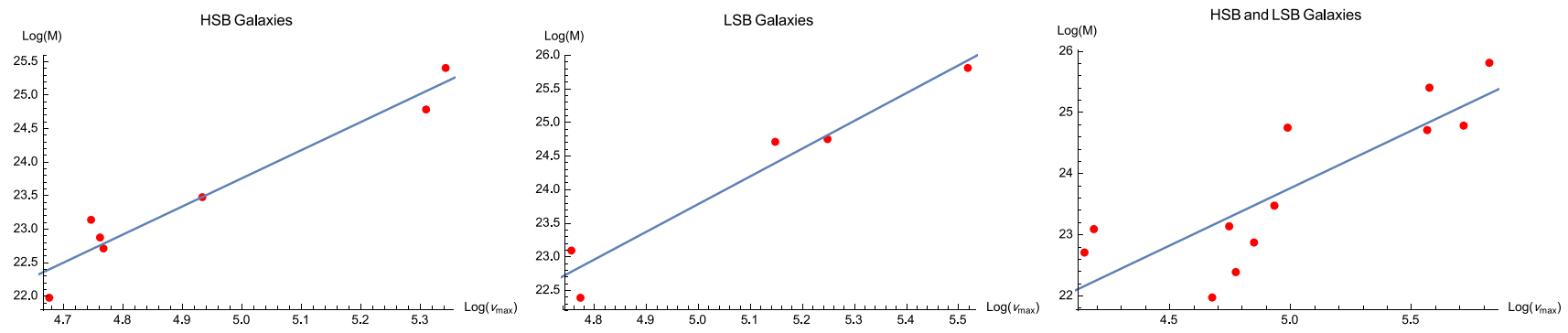

Fig. 2 The baryonic Tully-Fisher relation of 12 spiral galaxy samples [5]. The rotation velocity at quasi flat region $v_{q f}$ is given in the units of $\mathrm{km} / \mathrm{s}$ and mass is in units $10^{10} M_{\text {sun }}$. The red-dots are the results of our JBD-SVT model and they are obtained by calculating $v_{\max }$ for observational data of 12 spiral galaxies. The blue line is obtained via a least-square fit to the observational data in which

$a_{L S B}=4.13266, \quad b_{L S B}=3.1236$,

$a_{H S B}=4.19776, \quad b_{H S B}=2.77282$

and

$a_{L S B, H S B}=1.87782, \quad b_{L S B, H S B}=14.3757$.

One can see that the Eqs. (4.5) and (4.6) are in a good agreement with BTF given by (4.1) alone and slope of them are 4 approximately, while a combined LSB \& HSB given by (4.7) dose not satisfy the BTF and its slope reaches to some small values with respect to 4 . Difference of slopes with respect to the prediction in Ref. [44] as $3.5<a<4$, maybe resolved by regarding possibility observational errors, or by using more samples of the observational data, regarding cylindrical symmetry of the used spiral galaxies or by choosing other samples for the maximum circular velocity defined in Ref. [45]. Authors in most articles are used more than 100 sample of observed galaxies data to obtain slope of the empirical Tully Fisher relation. Since BTF is an empirical relation so the existence of the Baryonic Tully-Fisher relation may implies that the mass observed in baryons is the total mass [46] and it challenges the dark matter hypothesis.

\section{Concluding remarks}

The rotation curves of galaxies still remain as one of the profound challenges in the present day physics. In this paper we have considered an alternative view to the dark matter problem. In fact we used generalized Jordan-Brans-Dicke scalar-vector-tensor (JBD-SVT) gravity model to generate galaxy rotation curves where the time-like vector field $N_{\mu}$ is coupled non-minimally to the BD scalar field $\phi$ and the background metric. In weak field limits, we considered a powerlaw self-interaction potential for the vector field, which plays an important role to produce a repulsive Yukawa like metric potential. In fact we obtained that the vector field mass parameter is related to an effective cosmological constant which supports the acceleration of the universe in the large scale structure. In other word we obtained that in weak field limits metric around a galaxy under consideration behaves as modified Schwarzschild de Sitter space time. However this corrects galactic rotation curves by regarding the experimental observational data. The results were surprisingly the same as ones which are given in [12] but provided their physical basis are totally different. To test the observational consequences of the circular velocities in our model, we used the well-measured SPARC database [41] to fit the theoretical rotation curves predicted by JBD-SVT to the observational data. Our results are appropriately consistent with the observations. We then demonstrate that our results are in a good agreement with the baryonic Tully-Fisher relation for spiral galaxies. In short, the outlook of this work can be as follows: a vacuum sector of the Brans Dicke scalar tensor gravity in weak field limit which reduces to a Newtonian approach of the general relativity can not give a good fit to the galactic rotations curves (see blue dotes in Fig. 1). While a time-like dynamical self interacting vector field moving on the curved background metric of a galaxy can produce a good correspondence between theoretical results (see red-dotes in Fig. 1) of galactic circular velocities and observational data (see black dotes in Fig. 1). On the other hand, baryonic Tully Fisher relation confirms that one can describe galactic rotation curves correctly without using unknown cold dark matter and just with visible baryonic matter, using JBD-SVT theory. As further investigations one can study the solar system tests of the GBD-SVT theory, cosmological implications of the theory such as domain walls and study the form of modified virial theorem in the theory.

Acknowledgements The authors are grateful to the editor and anonymous referees for their valuable comments and suggestions which cause to improve the article for readers.

Data Availability Statement This manuscript has no associated data or the data will not be deposited. [Authors' comment: Because all experimental data which we used in this paper are available at the references $[5,40]$ which are addressed in the caption of the table 1 given in the paper.] 
Open Access This article is distributed under the terms of the Creative Commons Attribution 4.0 International License (http://creativecomm ons.org/licenses/by/4.0/), which permits unrestricted use, distribution, and reproduction in any medium, provided you give appropriate credit to the original author(s) and the source, provide a link to the Creative Commons license, and indicate if changes were made. Funded by SCOAP ${ }^{3}$.

\section{References}

1. G. Bertone, D. Hooper, J. Silk, Phys. Rep. 405, 279 (2005)

2. V.C. Rubin, W.K. Ford Jr., Astrophys. J. 159, 379 (1970)

3. V.C. Rubin, E.M. Burbidge, G.R. Burbidge, K.H. Prendergast, Astrophys. J. 141, 885 (1965)

4. J.W. Moffat, JCAP 0603, 004 (2006). gr-qc/0506021

5. R.H. Sanders, S.S. Mc Gaugh, Ann. Rev. Astron. Astrophys. 40, 263 (2002). arXiv:astro-ph/0204521

6. J.D. Bekenstein, Phys. Rev. D 71, 069901 (2005)

7. P.D. Mannheim, D. Kazanas, Astrophys. J. 342, 635 (1989)

8. J.W. Moffat, Phys. Lett. B 355, 447 (1995). gr-qc/9411006

9. B. Mashhoon, Ann. Phys. 16, 57 (2007)

10. B. Mashhoon, Phys. Lett. B 673, 279 (2009)

11. J.W. Moffat, V.T. Toth, Mon. Not. R. Astron. Soc. 395, 25 (2009)

12. J.R. Brownstein, J.W. Moffat, Astrophys. J. 636, 721 (2006). Astro$\mathrm{ph} / 0506370$

13. J.R. Brownstein, J.W. Moffat, Mon. Not. R. Astr. Soc. 367, (2006)

14. J.W. Moffat, V.T. Toth, Astrophys. J. 680, 1158 (2008)

15. J.W. Moffat, Found. Phys. 23, 411 (1993)

16. J.W. Moffat, Int. J. Mod. Phys. D 2, 351 (1993)

17. M.A. Clayton, J.W. Moffat, Phys. Lett. B 460, 263 (1999)

18. J.F.G. Barbero, E.J.S. Villasenor, Phys. Rev. D 68, 087501 (2003)

19. T. Jacobson, D. Mattingly, Phys. Rev. D 64, 024028 (2001)

20. J.F. Barbero, Phys. Rev. D 54, 1492 (1996)

21. C. Brans, R. Dicke, Phys. Rev. 124, 925 (1961)

22. H. Ghaffarnejad, Gen. Relativ. Gravit. 40, 2229 (2008)
23. H. Ghaffarnejad, Gen. Relativ. Gravit. 41, 2941 (2009)

24. H. Ghaffarnejad, Class. Quantum Gravit. 27, 015008 (2010)

25. H. Ghaffarnejad, J. Phys. 633, 012020 (2015)

26. H. Ghaffarnejad, E. Yaraie, Gen. Relativ. Gravit. 49, 49 (2017)

27. H. Ghaffarnejad, H. Gholipour. arXiv:1706.02904 [gr-qc]

28. C.M. Will, K. Nordvedt Jr., Astrophys. J. 177, 757 (1972)

29. K. Nordvedt Jr., C.M. Will, Astrophys. J. 177, 775 (1972)

30. R.W. Hellings, K. Nordvedt Jr., Phys. Rev. D 7, 3593 (1973)

31. M.A. Clayton, J.W. Moffat, Phys. Lett. B 477, 269 (2000)

32. B.A. Bassett, S. Liberati, C. Molina-Paris, M. Visser, Phys. Rev. D 62, $103518(2000)$

33. C.M. Will, Theory and Experiment in Gravitational Physics (Cambridge University Press, Cambridge, 1993). (revised version) arXiv:gr-qc/9811036

34. C. M. Will, Living Rev. Relativ. 9 (2006). http://www. livingreviews.org

35. E. Gaztanaga, J.A. Lobo, Astrophys. J. 548, 47 (2001)

36. R.D. Reasenberg et al., Astrophys. J. 234, 925 (1961)

37. Y.M. Cho, Phys. Rev. Lett. 68, 3133 (1992)

38. D. Giannios, Phys. Rev. D 71, 103511 (2005). gr-qc/0502122

39. W.J.G. de Blok, S.S. McGaugh, J.M. van der Hulst, Mon. Not. R. Astron. Soc. 283, 18 (1996)

40. Y. Sobuti, Astronom. Astrophys. 464, 921-925 (2007)

41. http://astroweb.case.edu/SPARC

42. F. Lelli, S.S. McGaugh, J.M. Schombert, Astron. J. 152, 157 (2016). arXiv:1606.09251 [astro-ph.GA]

43. J.W. Moffat, S. Rahvar, Mon. Not. R. Astron. Soc 436, 1439 (2013)

44. R.B. Tully, J.R. Fisher, Astron. Astrophys. 54, 661 (1977)

45. F. Lelli, S.S. McGaugh, J.M. Schombert, H. Desmond, Mon. Not. R. Astron. Soc. 201, 1 (2019). arXiv:1901.05966 [astro-ph.GA]

46. M. Milgrom, Astrophys. J. 270, 371 (1983) 